\title{
Speckle attenuation by adaptive singular value shrinking with generalized likelihood matching in optical coherence tomography
}

Huaiguang Chen

Shujun $\mathrm{Fu}$

Hong Wang

Hongli Lv

Caiming Zhang 


\title{
Speckle attenuation by adaptive singular value shrinking with generalized likelihood matching in optical coherence tomography
}

\author{
Huaiguang Chen, ${ }^{a}$ Shujun Fu, ${ }^{a, \star}$ Hong Wang, ${ }^{a, b}$ Hongli Lv, ${ }^{a}$ and Caiming Zhang ${ }^{c}$ \\ aShandong University, School of Mathematics, Jinan, China \\ bUniversity of South Carolina, Department of Mathematics, Columbia, South Carolina, United States \\ 'Shandong University, School of Computer Science and Technology, Jinan, China
}

\begin{abstract}
As a high-resolution imaging mode of biological tissues and materials, optical coherence tomography (OCT) is widely used in medical diagnosis and analysis. However, OCT images are often degraded by annoying speckle noise inherent in its imaging process. Employing the bilateral sparse representation an adaptive singular value shrinking method is proposed for its highly sparse approximation of image data. Adopting the generalized likelihood ratio as similarity criterion for block matching and an adaptive feature-oriented backward projection strategy, the proposed algorithm can restore better underlying layered structures and details of the OCT image with effective speckle attenuation. The experimental results demonstrate that the proposed algorithm achieves a state-of-the-art despeckling performance in terms of both quantitative measurement and visual interpretation. (๑) 2018 Society of Photo-Optical Instrumentation Engineers (SPIE) [DOI: 10.1117/1.JBO.23.3.036014]
\end{abstract}

Keywords: image denoising; optical coherence tomography; generalized likelihood matching; group sparsity; adaptive backward projection.

Paper 170758R received Dec. 5, 2017; accepted for publication Mar. 7, 2018; published online Mar. 28, 2018.

\section{Introduction}

Optical coherence tomography (OCT) is an emerging optical imaging technology that performs high-resolution, cross-sectional tomographic imaging of internal structures in biological systems and materials. ${ }^{1}$ It is a noninvasive imaging modality and plays an important role in medical clinical diagnosis and monitoring of diseases of human retina. ${ }^{2}$ However, it takes advantage of low-coherence interferometry, which inevitably introduces speckle noise. ${ }^{3}$ Speckle noise can mask image features, which severely degrades the OCT imaging quality for clinical diagnosis and analysis. Mathematically, to attenuate speckle noise can be formulated as an estimate problem, i.e., finding an approximation of an original image $x$ from its observed one $y^{2-4}$

$y=x \cdot n$,

where $n$ denotes the multiplicative speckle noise.

There are many approaches to solve this problem. Methods to reduce speckle noise of OCT images are mainly divided into two categories. The first type of method uses physical techniques, such as the frequency compounding, ${ }^{5}$ strain compounding, ${ }^{6}$ angular compounding, ${ }^{7}$ and spatial averaging, ${ }^{8}$ to address the noise problem before final image formation. However, this method is not easily adapted to standard commercial OCT systems, because it requires significant modifications of the hardware of existing imaging systems. The second type of method relies on postprocessing of images, such as methods based on total variation and diffusion equation, 2,3,9 wavelet decomposition, ${ }^{4}$ local statistical, ${ }^{10}$ self-similarity, and sparsitybased methods. ${ }^{11-17}$ A second-order total generalized variation model was introduced for image decomposition to remove speckle noise from OCT images. ${ }^{2}$ Gong et al. gave an algorithm with total variation regularization in $\mathrm{OCT}^{3}$ it realized sufficient speckle noise reduction with delicate edge preservation. Jian et al. described an algorithm based on shrinkage in the curvelet domain to attenuate speckles in OCT images. ${ }^{4}$ Bernardes et al. proposed a special method named nonlinear complex diffusion filter (NCDF). ${ }^{9}$ An algorithm for speckle noise reduction in OCT was proposed based on log-space general Bayesian estimation. ${ }^{10}$ These algorithms can reduce noise to a certain extent, but they can also lead to the loss of image details and consequently blur important image features.

In addition to the above-mentioned methods, more advanced algorithms are also used to remove the noise in OCT images. Fang et al. proposed the multiscale sparsity-based tomographic denoising (MSBTD) method. ${ }^{11}$ They learned a sparse representation dictionary for each high signal-to-noise (SNR) ratio images and utilized such dictionaries to denoise the lowSNR B-scans. Sparsity-based simultaneous denoising and interpolation improves MSBTD by utilizing sparse representation dictionaries constructed from previously collected datasets instead of high-quality images from the target imaging subject, which method is known as sparsity based simultaneous denoising and interpolation (SBSDI). ${ }^{17}$ Yu et al. presented a two-stage probability-based nonlocal means (PNLM) algorithm to effectively reduce speckle noise. ${ }^{12}$ Aum et al. proposed a nonlocal means filter with double anisotropic Gaussian kernels substituting for the conventional homogeneous kernel to remove speckle noise from OCT images. ${ }^{13}$ Thapa et al. proposed a speckle noise reduction method for OCT images called multiframe weighted nuclear norm minimization. ${ }^{16}$ These algorithms search similar image blocks with the common Euclidean distance, which 
may have a good effect on additive noise, but it was not ideal for the treatment of multiplicative speckle noise. ${ }^{18}$ The stable additive noise is independent of the optical signal, yet the speckle noise is related to the signal. In addition, for lack of adaptive filtering of different image features, these algorithms often lose some important image information and lead to blurred image details to some extent.

In order to solve these problems, we propose a new sparsitybased approach to restore OCT images: adaptive singular value shrinking method based on generalized likelihood similarity. The main contributions of our work include:

1. Block matching by the generalized likelihood similarity. Many repetitive patterns can be found in OCT images and many blocks are similar to a local one. Therefore, the nonlocal self-similarity prior is a very strong and successful one for image denoising. Considering the intensity and spatial correlation of OCT data, and the characteristics of speckle noise, we propose a generalized likelihood ratio criterion to look for similar blocks. Image blocks are first extracted from a noisy OCT image by an overlapping way. For each local block, in a search window, a similar group is assembled using a block-matching method based on the generalized likelihood similarity. These similar image blocks are respectively stretched into column vectors and then arranged in order, so that a highly correlated low-rank matrix can be formed. An adaptive singular value shrinking is carried out on the matrix, then restored image blocks are aggregated to obtain a denoised OCT image.

2. Adaptive backward projection of image features. In order to achieve better denoising performance, an iterative adaptive regularization technique is adopted, which gives different regularization parameters according to the structural features of OCT images. Since speckle noise has different effects on different structures of the image, we propose a self-adaptive backward projection function in the backward projection stage. The adaptive backward projection function can give different projection parameters according to different structural features, so that the image information can be well preserved.

The remainder of this paper is organized as follows. In Sec. 2, we introduce the detailed process of the proposed method. In Sec. 3, we show some experimental results of our method, and compare it with other methods to validate its efficacy. Finally, we conclude our work in Sec. 4.

\section{Proposed Method}

Speckle noise is signal dependent and has an impact on various structures in the OCT data. ${ }^{19}$ In addition, considering the correlation between the local block data of the OCT, we propose a bilateral sparse denoising algorithm. The sparse representation algorithms may be easily adapted by current users of commercial OCT imaging systems. ${ }^{17}$ As a bilateral sparse representation, ${ }^{20}$ singular value decomposition (SVD) provides highly sparser representation of image data, where bigger singular values mainly describe image structures, and smaller singular values are mainly related to speckle noise. Therefore, through singular value shrinking, we can separate the useful information of the OCT image from speckle noise. Adopting a new similarity criterion for block matching and an adaptive backward projection strategy, the proposed algorithm can restore better underlying layered structures and details of the image as shown in the following experiments. Figure 1 shows a block diagram of the proposed method, where $K$ represents the final number of iterations. In the rest of this section, the procedures of our proposed method will be described in detail.

\subsection{Generalized Likelihood Matching}

A simpler and more effective grouping of a mutually similar block can be realized by matching, and the formed group is a low rank and sparse matrix. Block matching is employed to find blocks that exhibit high correlation to a given reference one. That is achieved by pairwise measuring the similarity between the reference block and candidate block.

The proposed singular value shrinking method first estimates the denoised image block, then it estimates each image pixel included in multiple image blocks to restore the whole image by aggregating denoised image blocks. After overlapping image blocks are extracted from a noisy OCT image $y$, for a noisy image block $Y_{i}$ with size $W \times W$, block matching is needed to assemble a block group based on certain similarity criterion in a square $L \times L$ search window centered at $Y_{i}$. The reference block $Y_{i}$ and its $N$-most similar blocks denoted $Y_{j},(j=i, 1,2, \ldots, N-1)$ are chosen to construct a group matrix using each similar block as a column of the group matrix, and its corresponding block group matrix $\mathcal{Y}_{i}$ is formed by $\mathcal{Y}_{i}=\left[Y_{i}, Y_{1}, \cdots, Y_{N-1}\right]$. In the similar matrix, the corresponding columns from similar image blocks lead to lower rank of the similar matrix ${ }^{21}$ and, consequently, a highly sparse representation



Fig. 1 Flowchart of the proposed OCT image denoising algorithm. 
of image blocks by SVD. That is, the singular energy of the similar matrix concentrates on the first few bigger singular values, which benefits the separation of speckle noise from the noisy OCT image.

In order to adapt the proposed algorithm to the multiplicative speckle noise in Eq. (1), from the viewpoint of statistical inference, ${ }^{19}$ we elaborate a similarity criterion based on generalized likelihood ratio ${ }^{18}$ for image block matching

$$
G\left(Y_{i}, Y_{j}\right)=\frac{\sum\left[\sum\left(Y_{i} \circ Y_{j}\right)\right]}{\sum\left\{\sum\left[\left(Y_{i}+Y_{j}\right) \circ\left(Y_{i}+Y_{j}\right)\right]\right\}},
$$

where $G\left(Y_{i}, Y_{j}\right)$ is the similarity measurement between the estimated block $Y_{i}$ and its neighbor block $Y_{j} . \sum\left[\sum\left(Y_{i} \circ Y_{j}\right)\right]$ calculates the sum of all elements of the matrix $Y_{i}{ }^{\circ} Y_{j}$. Note that $Y_{i} \circ Y_{j}$ represents an elementwise product of two matrixes $Y_{i}$ and $Y_{j}$.

Through mathematical induction, one can know that no matter what size of blocks $Y_{i}$ and $Y_{j}$, when two blocks are the same, $G\left(Y_{1}, Y_{2}\right)$ has a maximum of $\frac{1}{4}$. The more similar two blocks are, the closer the value of $G\left(Y_{i}, Y_{j}\right)$ is to $\frac{1}{4}$. Therefore, we calculate the similarity between the reference block and the candidate block according to Eq. (2). Then, we select $N$ larger candidate blocks of $G$ as similar blocks of the reference block.

In general, the larger the size of the search window is, the bigger the number of similar blocks is. However, the bigger the number of similar blocks is, the worse the correlation between them is and the longer the running time is. Therefore, we empirically determine the number of similar blocks. For example, in this paper, we take the size of the search window $L$ to be 11 , and the number of similar blocks $N$ to be 40 .

\subsection{Singular Value Shrinking}

For the similar matrix $\mathcal{Y}_{i}$ from similar matching blocks, an SVD is used

$\mathcal{Y}_{i}=U_{i} \Sigma_{i} V_{i}^{T}$

where $U_{i}$ and $V_{i}$ are unitary singular matrixes, $\Sigma_{i}$ is a diagonal singular value matrix.

Considering bigger singular values mainly describe image structures while smaller singular values are mainly related to speckle noise, we denoise the OCT image with speckle noise by singular value shrinking

$\hat{\mathcal{X}}_{i}=U_{i} F_{i}\left(\Sigma_{i}\right) V_{i}^{T}$

where $\hat{\mathcal{X}}_{i}$ is the estimated image block, and $F_{i}\left(\Sigma_{i}\right)$ is a shrinking function on the diagonal matrix $\Sigma_{i}$.

Properly choosing the shrinking function is a very important technique for reliably estimating the underlying real image. Here, observing that bigger singular values characterize image structures more importantly, we shrink these singular values with smaller strength in proportion as their importance. ${ }^{22}$

In the soft-threshold shrinking framework, ${ }^{23}$ we choose the shrinking function as

$F_{i}\left(\Sigma_{i}\right)=\operatorname{diag}\left[\max \left(\Sigma_{i j}-\omega_{i j}, 0\right)\right]$,

where diag is a diagonal operator, and the $j$ 'th threshold value $\omega_{i j}$ is designed to be inversely proportional to the $j$ 'th singular value $\lambda_{i j}$ of the similar matrix $\mathcal{Y}_{i}$ $\omega_{i j}=\frac{c}{\lambda_{i j}+\varepsilon}$

where $c>0$ is a constant, and $\varepsilon=10^{-10}$ is to avoid dividing by zero.

\subsection{Aggregation}

By aggregating all the estimated image blocks, the whole image can be reconstructed. As a result of taking the neighbors of each block to construct a similar group, a single block might belong to several groups, and multiple estimates of this block can be obtained. Thus, we aggregate different estimates of this block to obtain a denoised version $\hat{x}$ of the noisy OCT image by simple averaging.

\subsection{Adaptive Backward Projection}

In order to achieve better denoising performance, an iterative regularization method is proposed by using the backward projection technique, ${ }^{24}$ which is an efficient trick using the residual image to improve the denoised result. The proposed method is an iterative process, where the aim of the backward projection is to retrieve lost information from the difference between the original noisy image and the last denoised image. Thus, in the current denoising process, a new noisy image is constructed by adding back some information eliminated in the last step to the last denoised image

$y^{(k+1)}=\hat{x}^{(k)}+\delta\left[y-\hat{x}^{(k)}\right]$,

where $y$ is the original noisy image, and $\hat{x}^{(k)}$ is the denoised result produced in the $k^{\prime}$ th iteration stage. $y^{(k+1)}$ is a new noisy image, which is used for the $(k+1)$ 'th iteration. $\delta \in(0,1)$ is a projection coefficient: when $\delta \rightarrow 0$ a loss of image

Algorithm 1 Adaptive singular value shrinking

1. Input: Noisy image $y$

2. Initialize $\hat{x}^{(0)}=y, y^{(0)}=y$

3. for $k=1: K$ do

4. Compute adaptive back projection parameter $\delta$.

5. Backward projection: $y^{(k)}=\hat{x}^{(k-1)}+\delta\left[y-x^{(k-1)}\right]$

6. for each block $Y_{i}$ in $y^{(k)}$

7. Find similar image blocks to form similar matrix $\mathcal{Y}_{i}$

8. SVD: $[U, \Sigma, V]=\operatorname{SVD}\left(\mathcal{Y}_{i}\right)$

9. Estimate threshold value vector $\omega_{i}$

10. Obtain the estimation: $\hat{\mathcal{X}}_{i}=U F_{i}(\Sigma) V^{T}$

11. end for

12. Aggregate $\hat{\mathcal{X}}_{i}$ to form denoised image $\hat{x}^{(k)}$

13. end for

14. Output: Estimated image $\hat{x}^{(K)}$ 
information tends to appear; when $\delta \rightarrow 1$ the $(k+1)$ 'th estimation tends to be the same as the first one.

In order to retrieve lost information dropped by the estimated result $\hat{x}^{(k)}$, a key issue is the determination of the projection coefficient $\delta$. Generally speaking, image features such as image edges and details are easy to be smoothed out because image denoising is a low-pass filtering process, removing some high-frequency components in the image.

Based on the above considerations, to preserve image information better we should mainly supplement image edges around layered structures rather than flat regions during the iteration. Therefore, the projection coefficient function $\delta$ is designed as follows:

$$
\delta= \begin{cases}\min \left[\frac{t}{M(i, j)+t}, s\right], & M(i, j) \geq t \text { and } \frac{M(i, j)-t}{M(i, j)} \geq s \\ \min \left[\frac{M(i, j)}{M(i, j)+t}, s\right], & M(i, j) \geq t \text { and } \frac{M(i, j)-t}{M(i, j)} \\ \min \left[\frac{M(i, j)}{t}, \xi\right], & 0 \leq M(i, j)<t\end{cases}
$$

where the parameter $t$ is the optimal gradient threshold of image edge obtained by the Otsu algorithm. ${ }^{25}$ On the OCT image, the local image gradient $M(i, j)$ at each point $(i, j)$ is calculated with the finite difference method in eight directions within a $3 \times$ 3 local window

$$
M(i, j)=\frac{\sum_{p=-1}^{1} \sum_{q=-1}^{1}|y(i, j)-y(i+p, j+q)|}{8} .
$$

The mean gradient of image is obtained by averaging all local gradients $M(i, j)$ in the image of size $m \times n$

$M_{\text {image }}=\frac{\sum_{i=1}^{m} \sum_{n=1}^{n} M(i, j)}{m \times n}$.

Moreover, the edge gradient of image $M_{\text {edge }}$ is the average of $M(i, j)$ satisfying $M(i, j) \geq t$.

$s$ is a threshold on image edge, which is defined as

$s=\frac{M_{\text {edge }}-M_{\text {image }}}{M_{\text {edge }}}$.

Obviously, $0<s<1$.

When $M(i, j) \geq t, y(i, j)$ is regarded as on an edge of the image. If $\frac{M(i, j)-t}{M(i, j)} \geq s$, then one can derive $M(i, j) \geq \frac{t}{(1-s)}$ : the gradient $M(i, j)$ is much larger than the optimal edge threshold $t$, and we think that the pixel $(i, j)$ is on a relatively stronger edge. And $\delta=\min \left[\frac{t}{M(i, j)+t}, s\right]$ is set to keep boundaries of the image, where $\delta$ decreases as the gradient becomes larger. Otherwise, if $\frac{M(i, j)-t}{M(i, j)}<s$, then one can derive $M(i, j)<\frac{t}{(1-s)}$, so we think the gradient $M(i, j)$ and the optimal edge threshold $t$ are relatively close, and the pixels are on relatively weak edge,



(b)



(d)

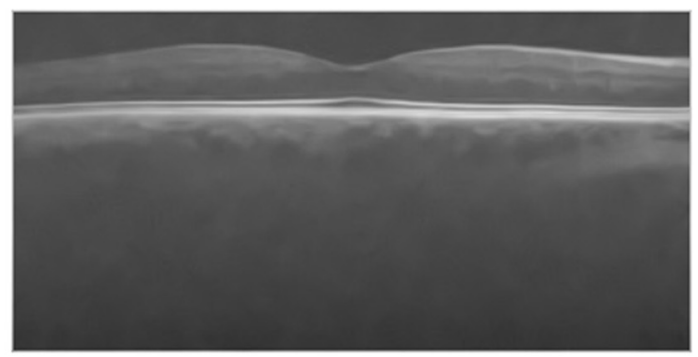

(f)

(e)

Fig. 2 Despeckling a noisy OCT image: (a) noisy image, (b) averaged image, results by (c) NCDF, (d) SBSDI, (e) PNLM, and (f) proposed methods, respectively. 
and $\delta=\min \left[\frac{M(i, j)}{M(i, j)+t}, s\right]$ is set, where $\delta$ increases as the gradient becomes larger, but it is always smaller than $s$. When $0 \leq M(i, j)<t, y(i, j)$ is regarded as in a flat region, and $\delta=$ $\min \left[\frac{M(i, j)}{t}, \zeta\right]$ is set to maintain image details, where $\zeta$ is an empirical parameter.

To summarize, the complete procedure of our proposed method is described in Algorithm 1.

\section{Experimental Results}

To evaluate the effectiveness of our proposed method, we compare its performance with some advanced denoising approaches: NCDF, ${ }^{9}$ SBSDI ${ }^{17}$ and PNLM. ${ }^{12}$ In our experiments, the parameters for NCDF, SBSDI, PNLM, and the proposed method for despeckling OCT images are selected for best quantitative metrics of filtered results. All methods are implemented using the
MATLAB ${ }^{\circledR}$ programming on a desktop computer with $2.83 \mathrm{GHz}$ CPU and 4 GB Memory.

Raw OCT images in the dataset from volunteers at the Duke Eye Center ${ }^{17}$ are employed to show despeckling effects of related methods. In the dataset, multiple repeated B-scan images are used to produce averaged images as referred noise-free images for image filtering comparison. In the experimental section, we selected the parameters empirically and kept them fixed. Note that, the optimal choices for such parameters should depend on the specific application. At the end of this section, we show the effect of the size and number of similar blocks on the experimental results.

We randomly select six OCT images from the dataset for following experiments of speckle noise removal. Due to limited space, we do not show them one by one, but only give a comparison of the denoising effect of one of the images. Among these, in Fig. 2, a noisy OCT image is despeckled by related

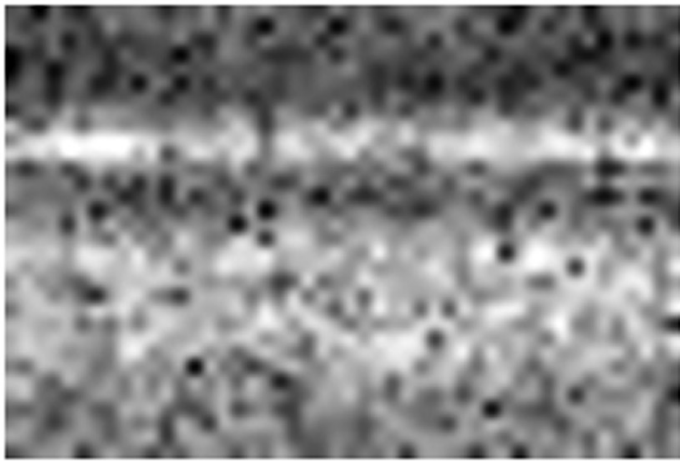

(a)

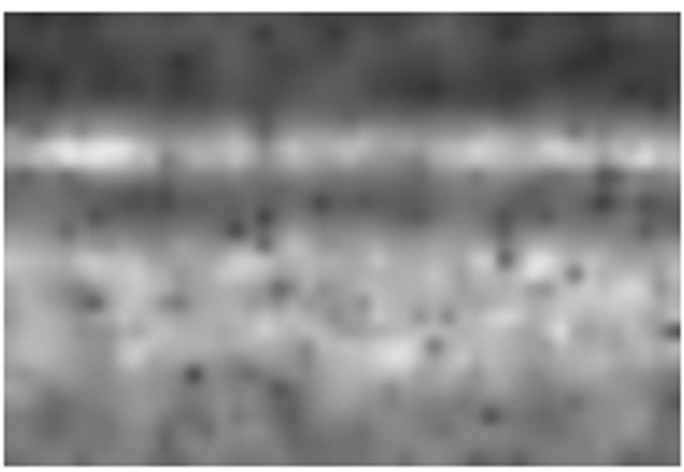

(c)

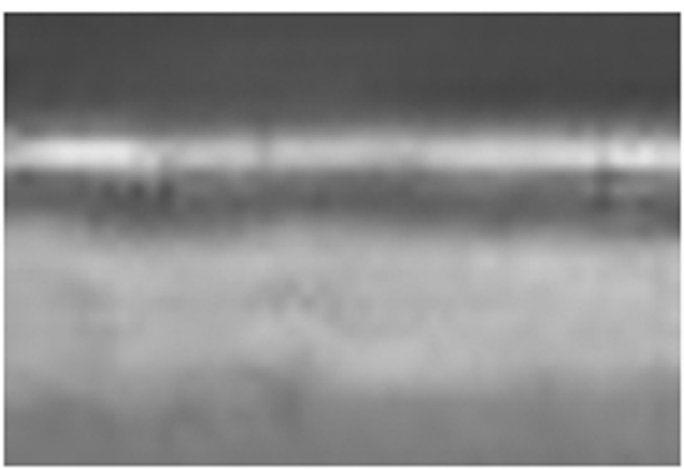

(e)

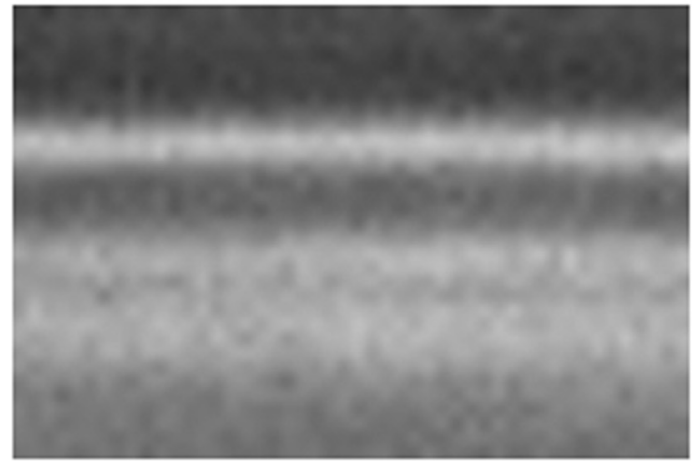

(b)

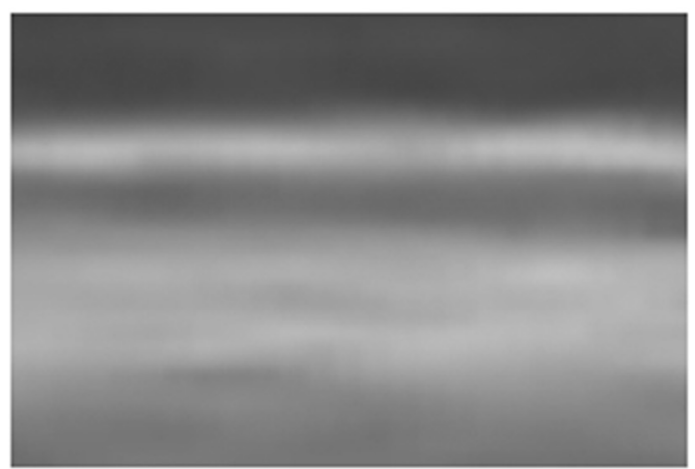

(d)

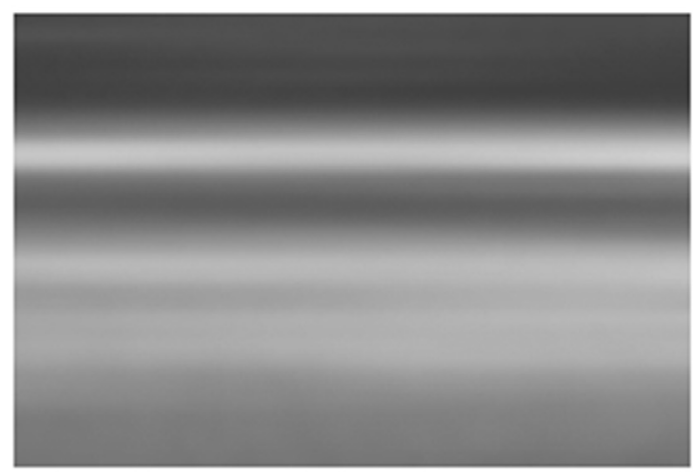

(f)

Fig. 3 Comparison of local layered structure in denoised OCT image: (a) noisy image, (b) averaged image, results by (c) NCDF, (d) SBSDI, (e) PNLM, and (f) proposed methods, respectively. 




(a)

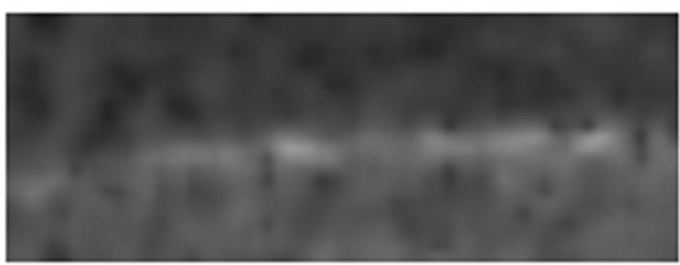

(c)



(e)

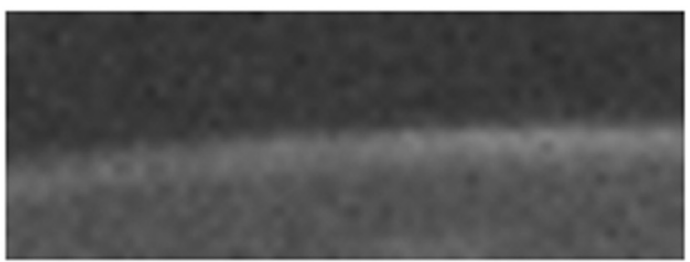

(b)

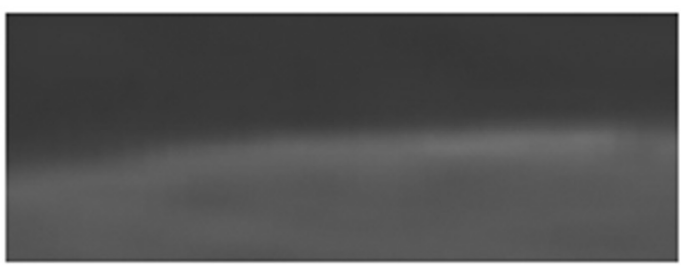

(d)

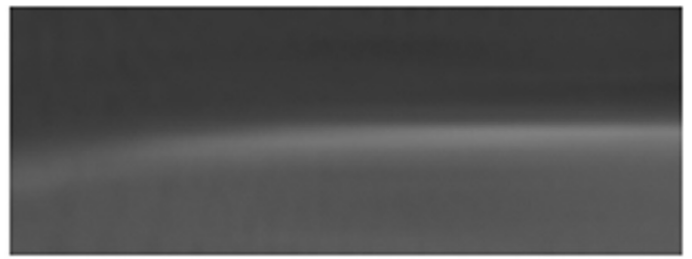

(f)

Fig. 4 Comparison of edge area in denoised OCT image: (a) noisy image, (b) averaged image, results by (c) NCDF, (d) SBSDI, (e) PNLM, and (f) proposed methods, respectively.

filtering methods, where we adopt $c=10, K=4, \zeta=0.36$, $L=11, W=15$, and $N=40$ in our method. For the sake of clarity, the local layered structure of the despeckled OCT image is furthermore shown in Figs. 3 and 4.

One can see that, for the observed OCT image, the layered structures and details are blurred heavily due to bigger speckle noise. By averaging multiple repeated images, speckle noise in the averaged image is effectively reduced though a little noise still remains all over the whole image, and the layered structures and details become relatively clearer than those of the noisy one. The NCDF method cannot remove noise effectively with most noise remained, and the layered structures and details are still blurry. Both SBSDI and PNLM methods reduce noise effectively, which make the layered structures clearer for one to observe. But these two methods produce nonsmooth layered structures bad for further image measurement and analysis in next clinic medical practice. Only the proposed method fully removes speckle noise preserving smooth layered structures and details. Sufficient speckle noise suppression makes layered structures observed clearly, which is helpful for next reliable edge detection and thickness measurement of layered structures.

To demonstrate the superiority of our algorithm, we analyze several quantitative metrics in addition to previous visual effect evaluations. The peak signal-to-noise-ratio (PSNR), ${ }^{18}$ feature similarity metric (SSIM), ${ }^{16}$ equivalent number of looks (ENL), ${ }^{3}$ and cross correlation (XCOR $)^{4}$ are calculated to evaluate despeckling results of different algorithms quantitatively. Apart from the whole image, some regions of interest marked in the green box in the noisy image in Fig. 2(a), which is also selected to demonstrate the performance of related algorithms for despeckling local layered structures. The averaged metrics from six test images are listed in Table 1 . In addition, the averaged metrics from five RIOs on Fig. 2(a) are shown in Table 2.
In Table 1, one can observe that our algorithm harvests the best PSNR, SSIM, and XCOR results, and the SBSDI algorithm provides the best ENL result. The reason for the smaller ENL of our algorithm may result from the denoising process, which may treat some speckle noise as the edge, but this situation has little effect on the overall effect of the algorithm. Therefore, for the whole OCT image, the proposed algorithm preserves more details and better suppresses speckle noise. In Table 2, our algorithm has the highest PSNR, SSIM, and XCOR results. Therefore, compared with other algorithms, our algorithm is the best in respect of the local layered structures denoising effect. The quantitative comparison further demonstrates the better recovery of both noisy RIOs and the whole OCT image by the proposed method due to its feature preserving suppression of speckle noise.

In addition to the previous metrics, we are looking at the complexity of the algorithm. The running time of all the methods is list in Table 3. It is worth noting that PNLM algorithm is written in $C$ or $C++$ language, but our algorithm is written in the pure MATLAB ${ }^{\circledR}$ language. We believe that the running time

Table 1 Averaged metrics of different denoising methods for six test images.

\begin{tabular}{lcccc}
\hline & PSNR & SSIM & XCOR & ENL \\
\hline NCDF & 26.5202 & 0.5017 & 0.9914 & 7.4843 \\
SBSDI & 30.3458 & 0.7067 & 0.9966 & $\mathbf{8 . 8 7 8 8}$ \\
PNLM & 29.6919 & 0.7085 & 0.9964 & 8.4714 \\
Proposed & $\mathbf{3 0 . 5 4 4 5}$ & $\mathbf{0 . 7 1 4 1}$ & $\mathbf{0 . 9 9 6 7}$ & 8.6624 \\
\hline
\end{tabular}

Note: The best results are marked in bold 
Table 2 Averaged metrics of different denoising methods for five RIOs.

\begin{tabular}{lcccc} 
& & & & \\
& PSNR & SSIM & XCOR & ENL \\
\hline NCDF & 26.7953 & 0.6464 & 0.9956 & 25.2256 \\
SBSDI & 29.5998 & 0.7902 & 0.9981 & 49.6632 \\
PNLM & 27.7702 & 0.7740 & 0.9980 & $\mathbf{6 2 . 1 3 9 7}$ \\
Proposed & $\mathbf{3 0 . 3 6 1 2}$ & $\mathbf{0 . 8 1 3 9}$ & $\mathbf{0 . 9 9 8 5}$ & 59.3741 \\
\hline
\end{tabular}

Note: The best results are marked in bold.

Table 3 Averaged running time (seconds) with different methods on six test image with size: $450 \times 900$.

\begin{tabular}{lcccc}
\hline Methods & NCDF & SBSDI & PNLM & Proposed \\
\hline Time (s) & $\mathbf{2 . 2 4}$ & 57.96 & 17.56 & 117.20 \\
\hline
\end{tabular}

Note: The best results are marked in bold.

of our algorithm can be greatly reduced when implemented with $C$ or $C++$. Moreover, the time of SBSDI does not include the running time of training the completed dictionary, which has higher computational complexity. Therefore, our algorithm will promote the application of OCT imaging in diagnosis and analysis of medical diseases.

Finally, through Tables 4 and 5, we give the influence of these empirical parameters on the experimental results of the proposed algorithm, where we only adjust one of the parameters keeping other parameters unchanged.

In Table 4, one can see that, as the block size increases, the PSNR and SSIM performance of the proposed method are expected to slightly improve. However, this improvement in performance comes with the decrease in the ENL. In addition, when the block size is $15 \times 15$, its denoising effect is relatively better and the shortest time is used. In Table 5, as the number of similar blocks increases the running time also gradually increases. Moreover, when the number of similar blocks is 40 the best denoising effect is achieved, and the structure of the image is well protected. Therefore, based on Tables 4 and 5, as well as experimental tests on other OCT data (not listed here), a compromise strategy is used to select the empirical parameters. For example, $15 \times 15$ is adopted as the block size and the number of similar blocks is 40 in our method.

Table 4 Comparison of metrics on different block sizes.

\begin{tabular}{ccccc}
$W$ & PSNR & SSIM & ENL & Time $(\mathrm{s})$ \\
\hline 11 & 31.6568 & 0.7307 & $\mathbf{1 0 . 4 7 0 2}$ & 156.09 \\
13 & 31.8466 & 0.7315 & 9.3741 & 150.43 \\
15 & 31.9813 & 0.7319 & 9.2984 & $\mathbf{1 1 3 . 4 3}$ \\
17 & 32.0745 & 0.7321 & 9.2124 & 1033.21 \\
19 & $\mathbf{3 2 . 1 4 8 4}$ & $\mathbf{0 . 7 3 2 4}$ & 9.1629 & 1756.27 \\
\hline
\end{tabular}

Note: The best results are marked in bold.
Table 5 Comparison of metrics on different numbers of similar blocks.

\begin{tabular}{lcccc}
$N$ & PSNR & SSIM & ENL & Time (s) \\
\hline 30 & 31.6696 & 0.7202 & 9.1627 & $\mathbf{1 0 1 . 7 0}$ \\
40 & $\mathbf{3 1 . 9 8 1 3}$ & $\mathbf{0 . 7 3 1 9}$ & 9.2984 & 113.44 \\
50 & 31.9773 & 0.7314 & 9.4150 & 140.95 \\
60 & 31.9705 & 0.7300 & 9.4322 & 162.34 \\
70 & 31.9631 & 0.7279 & $\mathbf{9 . 4 4 8 4}$ & 181.06 \\
\hline
\end{tabular}

Note: The best results are marked in bold.

\section{Conclusion}

An adaptive singular value shrinking based on the generalized likelihood ratio matching is studied in this paper. The proposed algorithm benefits from the highly sparse approximation of image data by the bilateral singular value shrinking, the proper similarity criterion for block matching, and the adaptive featureoriented backward projection strategy. Thus, in comparison with some advanced algorithms, the proposed algorithm obtains satisfactory visual effects and best image quality metrics. Finally, the proposed method can be expected of more successful applications of OCT imaging in medical diagnosis and analysis.

\section{Disclosures}

The authors have no relevant financial interests in this article and no potential conflicts of interest to disclose.

\section{Acknowledgments}

The research has been supported in part by the National Natural Science Foundation of China (61272239 and 61671276), the Science and Technology Development Project of Shandong Province of China (2014GGX101024).

\section{References}

1. D. Huang et al., "Optical coherence tomography," Science 254(5035), 1178-1181 (1991).

2. J. Duan et al., "Denoising optical coherence tomography using second order total generalized variation decomposition," Biomed. Signal Process. Control 24, 120-127 (2016).

3. G. Gong, H. Zhang, and M. Yao, "Speckle noise reduction algorithm with total variation regularization in optical coherence tomography," Opt. Express 23(19), 24699-24712 (2015).

4. Z. Jian et al., "Speckle attenuation in optical coherence tomography by curvelet shrinkage," Opt. Lett. 34(10), 1516-1518 (2009).

5. M. Pircher et al., "Speckle reduction in optical coherence tomography by frequency compounding," J. Biomed. Opt. 8(3), 565-569 (2003).

6. B. F. Kennedy et al., "Speckle reduction in optical coherence tomography by strain compounding," Opt. Lett. 35(14), 2445-2447 (2010).

7. N. Iftimia, B. E. Bouma, and G. J. Tearney, "Speckle reduction in optical coherence tomography by 'path length encoded' angular compounding," J. Biomed. Opt. 8(2), 260-263 (2003).

8. D. P. Popescu, M. D. Hewko, and M. G. Sowa, "Speckle noise attenuation in optical coherence tomography by compounding images acquired at different positions of the sample," Opt. Сотmun. 269(1), 247-251 (2007).

9. R. Bernardes et al., "Improved adaptive complex diffusion despeckling filter," Opt. Express 18(23), 24048-24059 (2010). 
10. A. Wong et al., "General Bayesian estimation for speckle noise reduction in optical coherence tomography retinal imagery," Opt. Express 18(8), 8338-8352 (2010).

11. L. Fang et al., "Sparsity based denoising of spectral domain optical coherence tomography images," Biomed. Opt. Express 3(5), 927-942 (2012).

12. H. Yu, J. Gao, and A. Li, "Probability-based non-local means filter for speckle noise suppression in optical coherence tomography images," Opt. Lett. 41(5), 994-997 (2016).

13. J. Aum, J. H. Kim, and J. Jeong, "Effective speckle noise suppression in optical coherence tomography images using nonlocal means denoising filter with double Gaussian anisotropic kernels," Appl. Opt. 54(13), D43-D50 (2015).

14. X. Zhang et al., "Spiking cortical model-based nonlocal means method for speckle reduction in optical coherence tomography images," $J$. Biomed. Opt. 19(6), 066005 (2014).

15. Z. Amini and H. Rabbani, "Optical coherence tomography image denoising using Gaussianization transform," J. Biomed. Opt. 22(8), 086011 (2017).

16. D. Thapa, K. Raahemifar, and V. Lakshminarayanan, "Reduction of speckle noise from optical coherence tomography images using multiframe weighted nuclear norm minimization method," J. Mod. Opt. 62(21), 1856-1864 (2015).

17. L. Fang et al., "Fast acquisition and reconstruction of optical coherence tomography images via sparse representation," IEEE Trans. Med. Imaging 32(11), 2034-2049 (2013).

18. C. A. Deledalle, L. Denis, and F. Tupin, "How to compare noisy patches? Patch similarity beyond Gaussian noise," Int. J. Comput. Vision 99(1), 86-102 (2012).

19. N. M. Grzywacz et al., "Statistics of optical coherence tomography data from human retina," IEEE Trans. Med. Imaging 29(6), 1224-1237 (2010).

20. W. Dong, G. Shi, and X. Li, "Nonlocal image restoration with bilateral variance estimation: a low-rank approach," IEEE Trans. Image Process. 22(2), 700-711 (2013).

21. J. Cai, E. J. Candes, and Z. Shen, "A singular value thresholding algorithm for matrix completion," SIAM J. Optim. 20(4), 1956-1982 (2010).

22. S. Gu et al., "Weighted nuclear norm minimization and its applications to low level vision," Int. J. Comput. Vision 121(2), 183-208 (2017).

23. S. Fu and C. Zhang, "Fringe pattern denoising via image decomposition," Opt. Lett. 37(3), 422-424 (2012).

24. S. Osher et al., "An iterative regularization method for total variationbased image restoration," SIAM Multiscale Model. Simul. 4(2), 460-489 (2005).

25. N. Otsu, "A threshold selection method from gray-level histograms," IEEE Trans. Syst. Man Cybern. 9(1), 62-66 (1979).
Huaiguang Chen received his BS degree from the School of Mathematics, Liaocheng University, China, in 2013, and his MS degree from the College of Mathematics from Qufu Normal University, China, in 2016. He is currently pursuing his PhD with the School of Mathematics, Shandong University, China. His research interests are medical image processing and image analysis, especially in image denoising and image enhancement.

Shujun Fu received his BS degree in power engineering and his MS degree in computational mathematics from Shandong University in 1990 and 1999, respectively, and his PhD in signal and information processing from Beijing Jiaotong University in 2009. He is currently a professor in the School of Mathematics, Shandong University. He has authored about 60 papers in important journals and conferences. His research interests include image processing, partial differential equations, numerical computing, medical imaging, image measurement, and target detection and recognition. He is also a peer reviewer on some important journals.

Hong Wang received his BS degree in power engineering and his MS degree in computational mathematics from Shandong University in 1982 and 1984, respectively, and his $\mathrm{PhD}$ in mathematics from the University of Wyoming, USA, in 1992. He is currently a professor in the School of Mathematics, University of South Carolina and Shandong University. His research interests include numerical approximation to differential/integral equations, scientific computations, and image processing.

Hongli Lv received his BS degree from the School of Mathematics and Statistics, Henan University, Kaifeng, China, in 2007, and his MS degree from the College of Mathematics and Information Science from Wenzhou University, Wenzhou, China, in 2015. He is currently pursuing his PhD in the School of Mathematics, Shandong University, China. His research interests include medical image processing and computer vision, especially image denoising and image segmentation.

Caiming Zhang received his BS and MS degrees in computer science from Shandong University in 1982 and 1984, respectively, and his $\mathrm{PhD}$ in computer science from Tokyo Institute of Technology, Japan, in 1994. He is currently a professor and the doctoral supervisor in the School of Computer Science and Technology, Shandong University. From 1997 to 2000, he has held visiting position with the University of Kentucky, USA. His research interests include CAGD, CG, information visualization, and medical image processing. 